\title{
CAHR: A Contextually Adaptive Home-Based Rehabilitation Framework
}

\author{
Ali Karime, Mohamad Eid, Haiwei Dong, Mohamad Halimi, Wail Gueaieb, and Abdulmotaleb El Saddik
}

\begin{abstract}
Home-based rehabilitation has evolved in recent years as a cost-effective and convenient alternative to traditional clinical rehabilitation. This has consequently created the need to design reliable assessment and adaptation mechanisms that are able to measure and analyze the patient's performance and to accordingly make proper adjustments that conform to the abilities of the patient during the training. This paper proposes a novel Context-based Adaptive Home-based Rehabilitation (CAHR) framework to optimize recovery based on patient's performance and ambient conditions. Adaptation is mathematically modeled to configure the rehabilitation task based on user's psychophysiological and environmental conditions. A prototype of the framework is tested for three weeks by two patients with wrist deficiencies. The results obtained are promising and indicate that the proposed framework may be very beneficial for home-based rehabilitation.
\end{abstract}

Keywords - Intelligent adaptation, rehabilitation framework, medical measurement, tangible user interface, cloud rehabilitation.

\section{INTRODUCTION}

Physical injury is a condition that affects the life of millions of people all around the world. Depending on the severity of the injury, patients normally undergo a rehabilitation regimen that aim to bring them back to pre-injury levels. The recent technological advancements have enabled physiotherapists to incorporate computers and a number of the state-of-the-art training devices into the rehabilitation training of their patients [1]. Computerized rehabilitation or virtual rehabilitation relies on video games, namely Virtual reality (VR) to enhance specific skills through playing [2]. The use of Virtual Environments (VE) in computerized rehabilitation has been shown to be a promising technology and was found to have potential benefits as an assessment and training tool [3-4]. Recently, virtual home-based rehabilitation (VHR) has evolved as a cost-effective and convenient alternative to traditional clinical rehabilitation. Researchers have developed various types of sensor-based rehabilitation systems that incorporate Virtual Reality games aimed to offer the patient an entertaining and beneficial training experience from the comfort of home [5-6]. This has consequently created the need to design reliable assessment and adaptation mechanisms that are able to measure and analyze the patient's performance and condition, and to accordingly make proper adjustments that conform to the abilities of the patient during the training.

Adaptivity attempts to maintain an appropriate level of challenge by dynamically adjusting game elements, such as the difficulty level and the environment within a game, according the player's performance [7]. Adaptation of digital games is an active area of research that focuses on using Artificial Intelligence (AI) to create Dynamic Adaptation Adjustment (DAA) models that fit the player's skills [8-9].The necessity of incorporating adaptation in rehabilitation related games becomes even more significant. It is important to keep the patient engaged and excited in VHR. However, it is very vital to ensure that the chances of getting re-injured because of requiring hard tasks that go beyond the patient's abilities are reduced to a minimum [10]. Furthermore, it is believed that effective adaptive systems can potentially shorten the recovery time since the training tasks are adjusted to efficiently address the deficiencies of the patient [11].

In this paper, we present CAHR, an adaptive virtual homebased rehabilitation framework that aims to offer the patient a proper in-home training that is optimized to his/her physical and psychophysiological conditions while taking into consideration the context of his or her surrounding environment. The major contribution of this work consists of proposing a novel adaptation model that suggests how the rehabilitation environment of the training tasks must be adjusted in order to match the patient's captured contextual information to the desired performance targets set by the therapist. A number of simulations are performed in order to validate its effectiveness and stability when dealing with various training scenarios. We use the tangible stress ball [12] and a game prototype developed based on the framework to evaluate the performance of two patients with wrist impairments who were trained with the game for two weeks. The rest of the paper is organized as follows. In Section II we review some of the related work accomplished in the field of adaptive computerized rehabilitation. In Section III we present the proposed CAHR framework and elaborate its merits. In Section IV we present the mathematical model of the adaptation engine and simulate its behavior. In Section V, we briefly present the framework prototype. In section VI, we reveal the experimental results conducted with two patients who trained with a prototype of the CAHR framework. Finally, in Section VII, we conclude by providing perspectives for future work.

\section{RELATED LITERATURE}

Despite its importance in virtual rehabilitation, only few researchers have proposed frameworks or designed systems that incorporate adaptation algorithms [13]. Rossol [14] proposed a framework for self-adjusting adaptive training in Virtual Environment (VR) rehabilitation games that is based on Bayesian Networks (BN). The framework's main goal was to help therapists probabilistically assess the patient performance in a complex range of several skill areas by providing 
meaningful discrete ranges. The $\mathrm{BN}$ network is modeled depending on the knowledge base of the clinician who customizes the network topology of the $\mathrm{BN}$ to include several numbers of skill areas (latents) and measured variables along with complex interaction between them. Alamri [15] developed an augmented-reality framework that adopts the concept of tangible objects as a means of interaction. Virtual objects are superimposed over a real environment set up, and the patient is required to perform a certain rehabilitation task by manipulating a real cup in the Augmented Reality (AR) environment. The framework includes two reaching exercises that are derived from daily life activity, such as moving a cup to a virtual cupboard. In [13], Pirovano presented a framework for self-adaptive games for home based-rehabilitation that adopts the fuzzy Logic and Bayesian adaptive methods as means of adaptation. In first algorithm, the fuzzy theory was used to analyze the correctness of the exercise by properly choosing an appropriate set of rules and constrains specified by the therapist. The second algorithm was built upon the Quest Bayesian adaptive approach [16] used in psychophysics to adapt a psychometric threshold considering the results of the previous trials. The main goal is to continuously adapt a parameter value so that it converges to a predefined threshold (defined by the therapist) that guarantees that a target performance level is met in a certain trial based on the patient's actual status. In [17], we presented a fuzzy-based adaptive framework for wrist rehabilitation that adjusts the intensity level of a software game depending on the patient's physical performance. The detected performance metrics are fed into a fuzzy logic adaptation (FLA) engine that evaluates the quality of performance and provide a crisp value that indicates how normal the performance of patient is for a particular task. The membership functions of the FLA were designed based on a set of performance thresholds which indicate the minimum level that a patient should attain in order to be assessed as healthy.

Ma [18] developed an adaptive VR game for post-stroke patients with upper extremity deficiencies that aim to encourage movement and improve their accuracy and speed. The game can be controlled through motion data captured by three sensors, Motion-Star Wireless, VR 1289 HMD, and the 5DT data glove that enable the detection of the position and orientation of the patient's hand. The game adaptation is achieved through a simple accuracy test function that provides an accuracy ratio between the number of successful and unsuccessful trials. Chen [19] introduced a media adaptation framework that can be used in a multi-model biofeedback system [20] for stroke patients undergoing arm rehabilitation. The adaptation model is based on a pre-trained Dynamic Decision Network (DDN) that predicts the patient's performance and suggests an online optimal adaptation decision to the rehabilitation team (e.g. therapists, doctors, etc.). The media adaptation in the framework refers to changes in audio and visual feedback within the virtual environment, as well as changes in the physical environment (e.g. table height).

In the related literature mentioned so far, the authors have relied mainly on the physical performance of the user to adapt the rehabilitation task or environment. Many studies have revealed that physiological conditions (e.g. fatigue and stress) psychological reactions (e.g. sadness or happiness), and environmental factors (e.g. ambient noise and lighting) can significantly impact the human's performance to properly accomplish various tasks [21-23]. Consequently, the effect of the physiological state of the user on the gaming performance has been explored by numerous researchers such as [24], [25], and [26]. In [27] the authors have investigated the possibility of using emotional data in adapting the difficulty of a game. This was done by asking 20 participants to play a modified version of a Tetris game which incorporates three different difficulty levels. Afterwards, two different analyses were conducted investigate how different levels of the game can affect the emotional state of the player and to validate that the users' states can be recovered from physiological signals only. The study concluded that different difficult levels can indeed elicit several emotional states and that game adaption according to player's emotion can maintain his or her engagement in the game. Even though the authors have validated the flow theory of games, no adaptive version of the Tetris game was implemented and was left as a future work. In [28], the authors explored the significance of the affect-based adaptation on the gaming experience of the user. The authors conducted two series of experiments that aim to compare the gaming experience of the players when playing with two versions of a Pong game. An affect-based version that can adapt depending on the emotional state on the user, and another version that adapts solely based on the player's performance. The results had shown that players had performed better with the affectbased version and that they had a better experience than playing with the performance-based version. However, the authors noted that an adaptation that relies on the physiological state may not be optimal and that the performance factor should be taken into account.

Based on the outcome of these studies and on the current related works in VHR , we conclude that an effective virtual rehabilitation framework should not mainly consider the patient's physical aspect when adapting the rehabilitation task, but also it should take into account his or her psychophysiological state and any environmental factors that may negatively affect the training experience. In addition, task adaptation should be applied in an online fashion (i.e. between tasks) rather than in offline mode (i.e. between sessions) so that the environment can be adjusted to fit the context of the patient in real time. Moreover, the adaptation of the environment must be based on the therapist's knowledge in the sense that it should enable him or her to set target performance goals tailored to the patient's capabilities. In our proposed framework, we address each of the aforementioned points in order to offer a training environment that is safe and effective for a home based rehabilitation. 


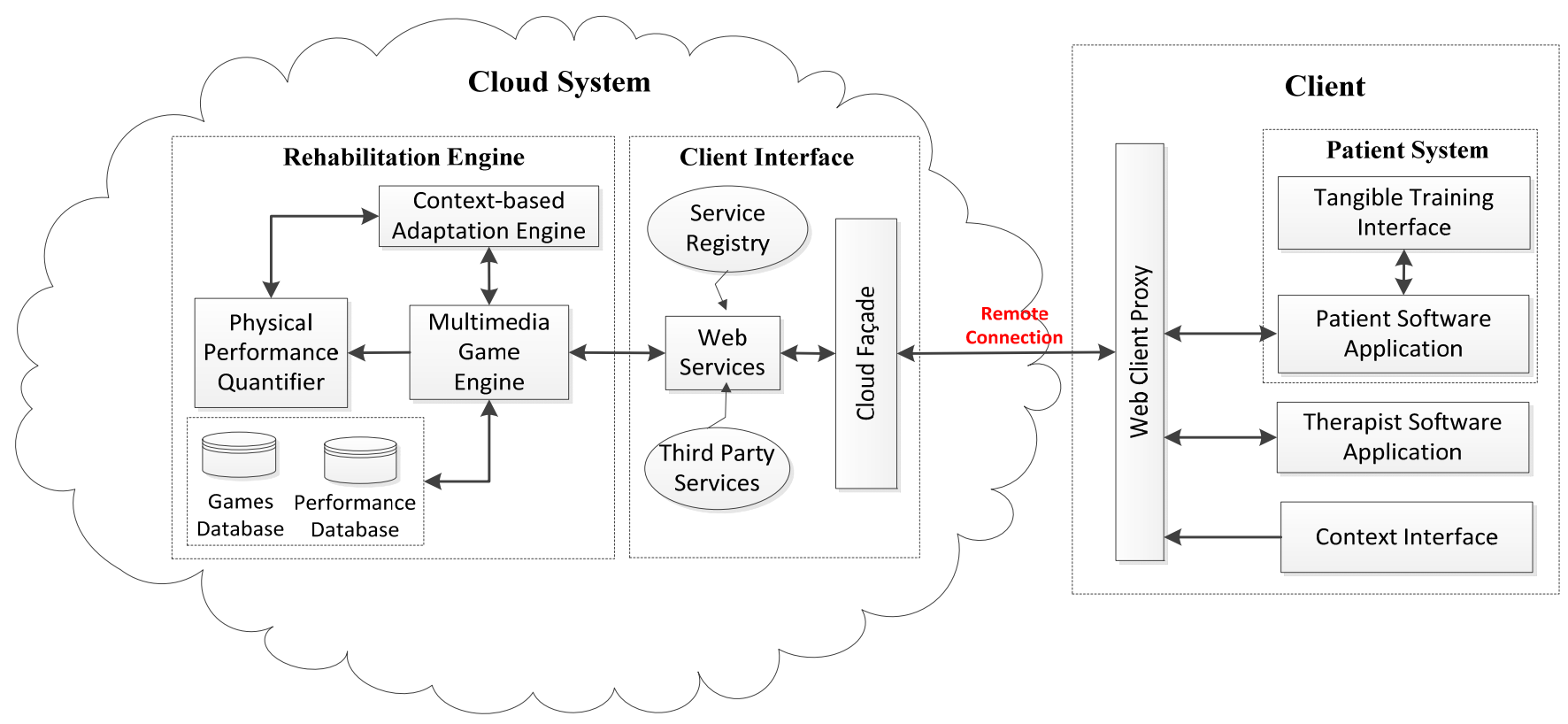

Fig.1. The proposed CAHR framework

\section{THE FRAMEWORK}

In a broader view, the framework shown in Figure 1 serves two types of users, the patient and the therapist. The patient can have a computerized home-based rehabilitation system that is simple to grasp, natural to be trained with, and performance informative. From the clinician's perspective, the framework helps the therapist to better understand the performance of the patient, get informed about his/her physiological and psychological state and the environmental conditions surrounding him or her, and to customize the adaptation of the virtual exercises.

The proposed model captures and utilizes the patient's physical performance, psychophysiological responses, and environmental conditions to provide an optimized adaptation for home-based rehabilitation. Therefore, it takes into consideration the various contexts that may hinder the patient to progress during the rehabilitation training. The model enables the clinician to select the contextual parameters that affect his/her patient and to define their correlations with the patient's ability to perform a task. This is done by assigning a weight between all of the context parameters. As a result, the model generates an adaptation matrix that defines how the psychophysiological responses and environmental conditions would be manipulated in order to improve the patient's quality of experience and to keep the patient more engaged in the rehabilitation training.

\subsection{The Client System}

The Client is a software component that provides various stakeholders (such as the patient, therapist, or a third party) access to the home-based rehabilitation system over the web. It is comprised of the Web Client Proxy, the Patient System, the Therapist Software Application, and the Context Interface.
The Web Client Proxy is a software application that provides services for retrieving, presenting and traversing web contents on the World Wide Web (HTML interpreter and SOAP for exchanging structured information via the Web). It also facilitates the communication between the Client System and the Cloud System.

The Patient System provides the patient with the means to interact with the system through the sensorized Tangible Training Interface or to communicate with the therapist via the Patient Software Application. Tangible Training Interfaces are non-conventional interfaces such as intelligent tangible devices that can detect the motion of the patient and provide haptic feedback if needed.

The Patient Software Application is the patient's door to the framework. It enables him or her to check information from the patient's treatment plan and displays the appropriate Virtual Reality games that are prescribed by the remote therapist.

The Therapist Software Application enables the clinician to monitor the patient's performance by retrieving information extracted during the game sessions. In addition, it allows the therapist to customize the rehabilitation task by offering him/her the capability to define a number of performance thresholds that best fit the physical and physiological conditions of the patient.

The Context Interface provides the rehabilitation framework with information related to the patient's context. This interface can be any of the hardware and/or software components that enable the detection of the physiological, psychological or environmental context. An example of such interface is a watch, a light sensor, a heart rate sensor, a brainwave headband, etc. 


\subsection{The Cloud System}

The Cloud System is composed of the Client Interface and the Rehabilitation Engine. It is an independent entity that manages various system functionalities (such as adaptation) and store relevant data (such as games and patient's performance data). The Cloud System can be hosted by an authorized entity to provide home-based rehabilitation services. The main advantage of adopting a cloud system architecture is that it provides to the patient the ability to train from anywhere at any time while keeping his performance records.

\subsection{The Client Interface Module}

The Client Interface module is located at the Cloud and provides abstract interfacing to individual clients. The client is typically located in a physically remote place. The Client Interface is composed of the Web Services, Service Registry, Third Party Services, and the Cloud Façade.

The Web Services is a component that hosts a pool of web services which provide various functionalities for both the patient and the therapist such as a web service to retrieve patient's performance information and provide a proper display.

The Service Registry enables clients to discover and access web services by storing information about the services functionalities as well as their interfaces (how to access the web services).

The Third Party Services are services that are hosted by third party service providers to provide assistive functionalities (such as certain type of signal processing or graphics display web services).

The Cloud Façade has a similar functionality as the Web Client Proxy. It is a software application that implements HTML interpreter and SOAP messenger for exchanging structured information with the client.

\subsection{Rehabilitation Engine}

The Rehabilitation Engine is the unit where all the performance data is analyzed and the game state decisions are made. It is comprised of the Physical Performance Quantifier, Context-based Adaptation Engine, Multimedia Game Engine, Game Database, and the Performance Database.

The Physical Performance Quantifier ( $P P Q$ ) aims to assess the Quality of Physical Performance ( $Q P P$ ) based on the physical kinematics captured during the rehabilitation training. The main goal is to provide the rehabilitation framework and the therapist with a crisp value that reflects how well or bad the patient is able to achieve a particular task from a physical perspective. To achieve this goal, the patient's physical performance is evaluated based on the Fuzzy Logic (FL) theory that is distinguished by its capability of reasoning in a human-like manner. The membership functions of the Fuzzy Inference Engine are constructed based on the set of benchmark kinematics which reflects the thresholds that a patient should achieve in order to be assessed as improving. Since we will be testing our framework with patients suffering from wrist impairments, the membership functions were constructed based on the norms found in [12]. A thorough details about this module can be found in [16].

The Context-based Adaptation Engine ( $C A E$ ) constitutes the core of the rehabilitation framework since it controls the intensity levels of the tasks and the multimedia content of the rehabilitation environment (e.g. recommendation messages, music, etc...). The complete details of this engine are given in the subsequent section.

The Multimedia Game Engine formulates and load the proper rehabilitation task for the patient based on the therapist's constraints, the patient's context, and the adaptation decisions made by the Context-aware Adaptation Engine. In addition, the engine provides multimedia feedback in the form of recommendations and vibro-tactile haptic feedback to the patient depending on his/her training performance and the game's state. The recommendations are shown in the form of text messages on the patient's screen. These messages are triggered based on the therapist's customization once a particular threshold is surpassed. For instance, the system might recommend the patient to take a 10 minute break if his stress level is really high. On the other hand, the engine sends vibro-tactile commands to the Training Interface Module upon achieving a particular goal during the task in order to make the rehabilitation session more entertaining and encouraging.

The Game Database stores information regarding the rendering and the configuration of the various rehabilitation games incorporated in the framework (such as the games graphics and default settings).

The Performance Database stores the performance and motion data captured throughout the rehabilitation training. This includes the raw data captured using the tangible interface, performance parameters (such as grasping angle/velocity, jerkiness, tremor, etc.).

\section{THE CONTEXT AWARE ADAPTATION MODEL}

We propose a context-aware adaptation model that considers the context of the patient and the therapist performance thresholds to properly adjust the tasks within a rehabilitation session so they fit the physical, physiological, psychological abilities of the patient while considering at the same the environmental condition.

\subsection{Rehabilitation Correlation}

Three matrices define the proposed model: the Measured Conditions Matrix ( $M$ ), the Desired Conditions Matrix ( $D$ ), and the Correlation Matrix ( $C$ ). The output of the mathematical model is the Adaptation Coefficients Matrix $(\alpha)$. Note that $\alpha_{i}$ represents the significance of the $i^{\text {th }}$ condition towards the patient's performance, which can also be used to adjust the various conditions to optimize the rehabilitation process.

The patient's performance in the home-based rehabilitation system is modeled using the Measured Conditions Matrix ( $M$ ) as shown in Equation 1: 


$$
M=\left[M_{1}, M_{2}, \ldots, M_{N}\right]^{T}
$$

where $M_{i}$ is the measured index for the $i^{\text {th }}$ condition and $N$ is the number of conditions to be considered in the adaptation model. The Desired Conditions Matrix $D$ is defined according to Equation 2:

$$
D=\left[\alpha_{1} \times D_{1}, \alpha_{2} \times D_{2}, \ldots, \alpha_{N} \times D_{N}\right]^{T}
$$

where $D_{i}$ is the desired index for the $i^{\text {th }}$ condition and $\alpha_{i}$ is the adaptation coefficient for the $i^{\text {th }}$ condition. The Desired performance index $D_{i}$ is set by the therapist to setup the optimal conditions for the patient to perform a rehabilitation task.

The Correlation Matrix $C$ (Equation 3) is a $N \times N$ matrix that describes the mutual correlation between any two conditions. For instance the element at $i^{\text {th }}$ row and $j^{\text {th }}$ column describes the correlation between the $i^{\text {th }}$ and $j^{\text {th }}$ conditions (such as the correlation between stress and fatigue). Note that the therapist defines the correlation matrix $C$ for every patient in order to personalize the model performance according to the patient's particular preferences and needs.

$$
C=\left[\begin{array}{cccc}
C_{i_{1} j_{1}} & C_{i_{1} j_{2}} & C_{i_{1} j_{3}} \ldots & C_{i_{1} j_{N}} \\
C_{i_{2} j_{1}} & C_{i_{2} j_{2}} & C_{i_{2} j_{3}} \ldots & C_{i_{2} j_{N}} \\
\vdots & \vdots & \vdots & \vdots \\
C_{i_{N} j_{1}} & C_{i_{N} j_{2}} & C_{i_{N} j_{3}} \ldots & C_{i_{N} j_{N}}
\end{array}\right]
$$

\subsection{Context-Aware Adaptation}

The ultimate goal of the proposed model is to match the Measured Conditions Matrix ( $M$ ) and the Desired Conditions Matrix ( $D$ ) by tuning the Adaptation Coefficients Matrix $(\alpha)$, which is the output of the adaptation model. As the adaptation coefficient $\alpha_{i}(1 \leq i \leq N)$ is unknown, an estimate model is defined according to Equation 4.

$$
M(t)=C D(t)=C\left[\begin{array}{c}
\alpha_{1} D_{1} \\
\vdots \\
\alpha_{N} D_{N}
\end{array}\right]
$$

Now the updated Measured Conditions Matrix ( $\hat{M}$ ) is computed by multiplying the Correlation Matrix $(C)$ by the estimated Desired Condition Matrix $(\hat{D})$ as shown in Equation 5.

$$
\hat{M}(t)=C \hat{D}(t)=C\left[\begin{array}{c}
\hat{\alpha}_{1} D_{1} \\
\vdots \\
\hat{\alpha}_{N} D_{N}
\end{array}\right]
$$

The idea is to update the adaptation coefficient $\hat{\alpha}_{i}(1 \leq i \leq N)$ by using the online data. The estimated Desired Condition Matrix $(\hat{D})$ can be obtained by minimizing the total error between the measured and the estimate model in the form of least-squares, as expressed in Equation 6.

$$
\min \int_{0}^{t} e^{\lambda\|M(r)-C \hat{D}(t)\|_{2}} d r
$$

where $\lambda>0$ is the forgetting factor, giving a weight of importance for the current measurement based on previous measurements. In other words, the forgetting factor $(\lambda)$ represents the weight of the history of patient's performance on the adaptation of the next rehabilitation task. For example, the larger the value of $\lambda$, the faster the adaptation occurs as the history of performance has less weight and thus the adaptation decision is mostly based on the current performance. This parameter can be defined by the therapist to realize a more personalized adaptive home-based rehabilitation system. Now, the update of $(\hat{D})$ can be derived as shown in Equation 7 .

$$
\dot{\hat{D}}=-P C^{T}(\hat{M}-M)=-P C^{T}(C \hat{D}-M)
$$

where $(P)$ is defined as the Gain Matrix and is defined according to Equation 8.

$$
P=\left(\int_{0}^{t} C^{T} C d r\right)^{-1}
$$

In order to update the gain matrix $(P$ ) without the need to do matrix inversion, the property described in Equation 9 has been utilized

$$
\frac{d}{d t}\left(P P^{-1}\right)=\dot{P} P^{-1}+P \frac{d}{d t}\left(P^{-1}\right)
$$

where

$$
\dot{P}=\frac{d P}{d t}=\frac{d}{d t}\left(\int_{0}^{t}\left(C^{T} C d r\right)^{-1}\right)
$$

Now, since $\frac{d}{d t}\left(P P^{-1}\right)=0$, we get

$$
\frac{d}{d t}\left(P P^{-1}\right)=\dot{P} P^{-1}+P \frac{d}{d t}\left(P^{-1}\right)=0
$$

therefore, this yields

$$
\dot{P}=-P \frac{d}{d t}\left(P^{-1}\right) P=-P C^{T} C P
$$

Finally at the next iteration $\dot{P}_{N e w}, P_{N e w}, \dot{\hat{D}}_{N e w}$ and $\hat{D}_{N e w}$ can be updated using Equations 13, 14, 15 and 16 respectively.

$$
\dot{P}_{\text {New }}=\lambda P_{\text {Old }}-P_{\text {Old }} C^{T} C P_{\text {Old }}
$$




$$
\begin{aligned}
& P_{\text {New }}=P_{\text {Old }}+K_{g} \dot{P}_{\text {New }} \\
& \dot{\hat{D}}_{\text {New }}=-P_{\text {New }} C^{T}\left(C \hat{D}_{\text {Old }}-M\right) \\
& \hat{D}_{\text {New }}=\hat{D}_{\text {Old }}+K_{u} \dot{\hat{D}}_{\text {New }}
\end{aligned}
$$

where $K_{g}$ and $K_{u}$ are constants that yield faster convergence.

Knowing the $\hat{D}$ and $D$ matrices, the adaptation coefficients can be computed according to Equation 17.

$$
\left[\begin{array}{c}
\hat{\alpha}_{1} \\
\vdots \\
\hat{\alpha}_{N}
\end{array}\right]=\left[\begin{array}{c}
\hat{D}_{1} / D_{1} \\
\vdots \\
\hat{D}_{N} / D_{N}
\end{array}\right]
$$

Notice that if $\hat{D}_{i} / D_{i} \approx 1$, this would mean that the patient has already achieved the target level of performance for parameter $i$.

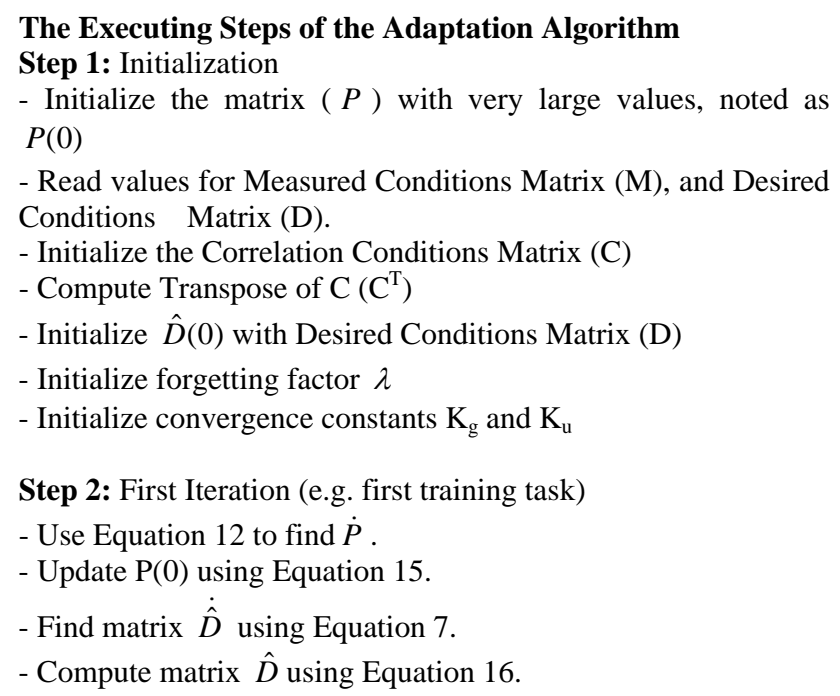

Step 3: Iterations Afterwards (e.g. second training task until the end of the training)

Loop while $\hat{D} / D<$ Threshold

- Find $\dot{P}_{N e w}$ using Equation 13.

- Update $P_{N e w}$ using Equation 14.

- Compute $\dot{\hat{D}}_{N e w}$ using Equation 15.

- Update $\hat{D}$ using Equation 16.

Find $\hat{\alpha}$ using Equation 17.

Here, Threshold is a value defined by the therapist.

Fig. 2. The execution procedure of the adaption algorithm

\subsection{Simulation Setup}

In this section, we apply different performance scenarios to our proposed adaptation model. The aim of these simulations is to test the behaviour of the proposed model in coping with the various performances that may be encountered during the training.

The CAHR's Context-based Adaption model is implemented in C\# and the experiments are run on a Pentium 4 machine with 4 GB RAM. We have chosen 4 contextual parameters as the measured elements in the matrix $M$ where the first element is the quality of physical performance ( $Q P P)$, the second is room's luminance, the third is stress, and the fourth is fatigue. It is worth noting that these contextual elements were chosen for testing purposes only. It is up to the therapist to choose the parameters that he or she thinks that they affect his or her patient the most. By adding or removing parameters, the therapist can govern the way adaptation should occur. For instance, if only $Q P P$ is chosen, then the adaptation engine would adjust the next rehabilitation task based on the physical performance only. Otherwise, if omitted, adaptation would rely more on the other parameters defined by the therapist.

The values in matrix D were randomly chosen. These values are the desired or the maximum values that should be achieved or allowed by the end of the training. These values were chosen as follows: the first element is the desired $Q P P$, the second is the desired luminance, the third is the maximum allowable stress level, and the fourth is the maximum allowable fatigue level. In addition, the effect of each element on the other parameters was chosen randomly as shown in the correlation matrix C. Figure 2 shows the execution procedure of the adaptation model.

$$
\begin{aligned}
M & =\left[\begin{array}{llll}
0.6 & 0.8 & 0.7 & 0.5
\end{array}\right]^{T} \\
D & =\left[\begin{array}{llll}
0.9 & 0.8 & 0.75 & 0.7
\end{array}\right]^{T} \\
C & =\left[\begin{array}{cccc}
1 & 0.7 & 0.4 & 0.5 \\
0.7 & 1 & 0.3 & 0.2 \\
0.4 & 0.3 & 1 & 0.3 \\
0.5 & 0.2 & 0.3 & 1
\end{array}\right]
\end{aligned}
$$

\subsection{Simulation Results}

Three simulation cases are considered: linear, logarithmic, and sinusoidal.

\section{A. Case 1: Linear Improvement}

In this scenario, we assume that the patient's performance is improving almost at the same amount every day. The results are shown in Figure 3 for a linearly increasing simulation. The adaptation coefficients have been tuned in to match the desired and measured conditions. Furthermore, the forgetting factor $(\lambda)$ is shown to have a significant impact on the speed of 
adaptability. An increase of the forgetting factor $(\lambda)$ results in a faster convergence of the measured conditions towards the desired conditions.

\section{B. Case 2: Logarithmic Improvement}

In this scenario, we assume that the patient's performance was initially improving very fast (e.g. on the first 5 days of training), and then the improvement became much slower over time. Therefore, the patient's performance is modeled as increasing logarithmically as function of the rehabilitation task iterations. The results are shown in Figure 4 where again the measured conditions have converged to the desired conditions. Same impact of the forgetting factor $(\lambda)$ has been experienced in this case.

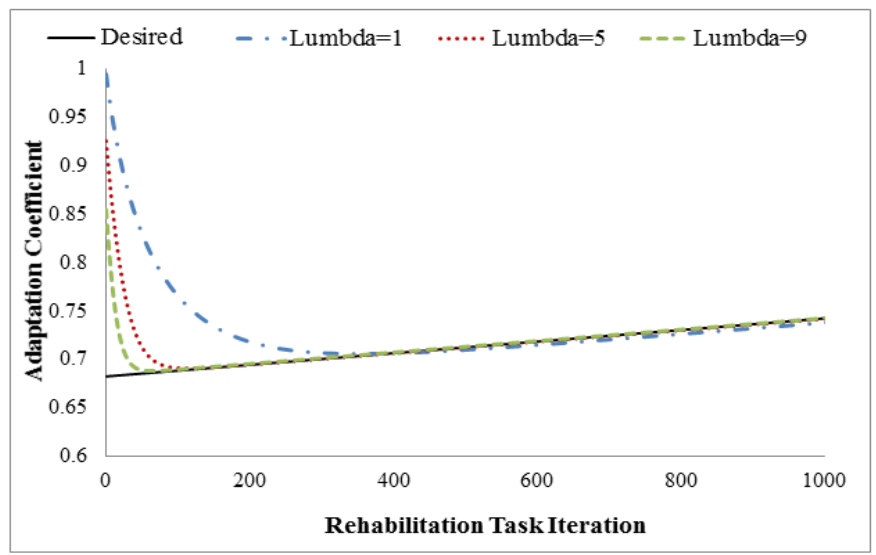

Fig. 3. Adaptation when patient's performance is a linearly increasing function

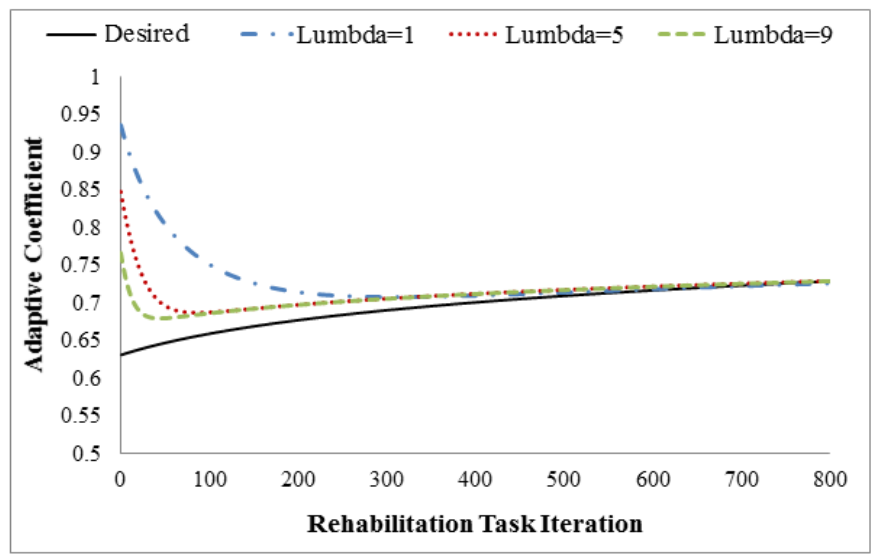

Fig. 4. Adaptation when patient's performance is a logarithmic function

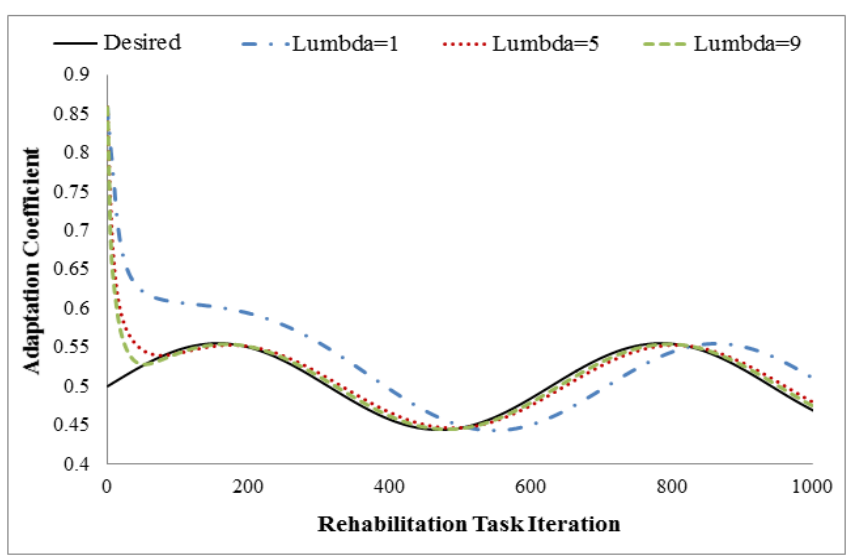

Fig. 5. Adaptation when patient's performance is a sinusoidal function

\section{Case 3: Sinusoidal Improvement}

In this last case, we assume that the patient's performance is fluctuating from time to time. For example, the patient might show an improvement on the first two days of training and then deterioration on the next two days. Thus, the performance is modeled as oscillating over the rehabilitation task iterations. The objective of the scenario is to evaluate the ability of the adaptation model to cope with fluctuations in the patient's performance. The results are shown in Figure 5 where the measured conditions converged to the desired conditions, except when the forgetting factor $(\lambda)$ is equal to 1 . The condition $(\lambda=1)$ implies that the adaptation model has experienced a latency to cope with higher frequency changes in the patient's performance. Therefore, a conclusion can be drawn from this simulation result that a patient with a fluctuating performance should be assigned a higher value of the forgetting factor $(\lambda)$ to make sure the adaptive model can cope and respond to abrupt changes in the patient's performance.

\section{FRAMEWORK PROTOTYPE}

We have implemented a software game based on the components shown in the Rehabilitation Engine module of the proposed framework. The "Cup \& Plate" is a special game designed to enable training of the wrist. The game was developed using the Java programming language. The Java swing library was used to create the 2-dimensional graphics, while the RxTx library was used for the serial communication with the digital stress ball. The Physical Performance Quantifier ( PPQ ) was implemented with the Xfuzzy software and then the resulting Fuzzy Inference System (FIS ) was exported to a Java Class. The reader can refer to [17] for more detailed information about the implementation of the $P P Q$.

We have used the digital stress ball [12] as the training interface. The ball was designed using a 6 DoF Inertial Measurement Unit (IMU) that is comprised of a 3-axis $A D X L$ 335 accelerometer and an ITG 3200 gyroscope that were attached on the ball's top surface. We have attached a triple axis MAG3110 Magnetometer to the lower surface of the ball. The data fusion of the 3 sensors allows the detection of the 
three motions of interest (pitch, roll, and yaw). Tilt and drift errors were compensated using a fusion filter that was deployed on a 16 MHZ Arduino Pro-mini 328. A 100 Lbs FlexiForce force sensor was properly attached to go under the palm of the users. Figure 6 shows a screen shot of the game and a picture of the stress ball. It is worth noting that the Cloud system was not implemented within the framework. We have rather stored all the data on the local database created on the hard drive of the laptop used during the training.

In order to play the game, the patient has to rotate his/her wrist on the Supination or Pronation motion to reach a cup which angular position is determined by the adaptation engine of the framework. Once the patient reaches the cup, he/she has to exert pressure on the stress ball in order to grab it. Afterwards, the patient should drag the cup back to the plate and drop it by simply releasing his or her grip force.

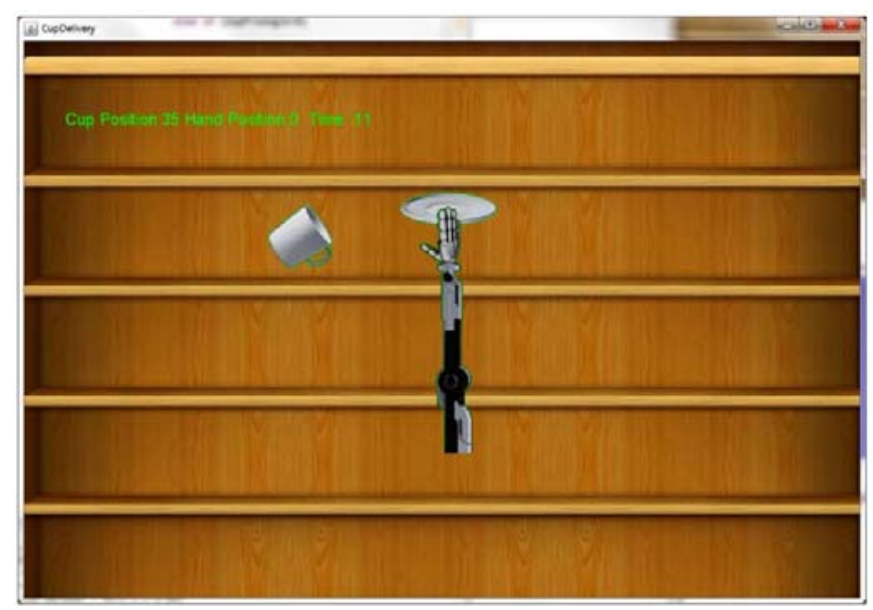

(a)

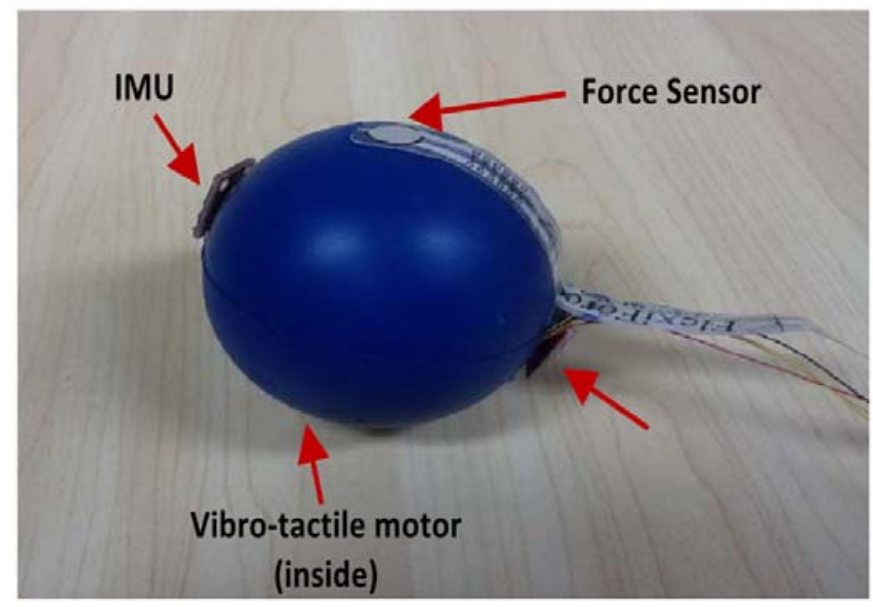

(b)

Fig. 6. The framework-based rehabilitation system: (a) The Cup \& Plate game and (b) the digital stress ball interface [12].

\section{PILOT STUDY}

The aim of the pilot study is to examine the impact of the proposed Context-based Adaptation model on the rehabilitation outcome of patients suffering from wrist deficiencies. In other words, we wanted to explore if the adaptation decisions made by this Context-based Adaptation module can really help the patient acquire suitable training levels that may enhance his or her abilities, and therefore bring him/her to a reasonable recovery state after training with the proposed framework.

\subsection{Patient Information}

Two right-hand dominant males, ages 35 and 50, participated in this study. The first patient labeled ML suffered a fracture at the forearm while the second patient labeled AS had a hand fracture. Both patients had deficiencies in performing Supination and Pronation rotations and were unable to achieve the full Ranges of Motion on the two motions. In addition, both patients were at the early stages of a physical rehabilitation program when they participated in the tests.

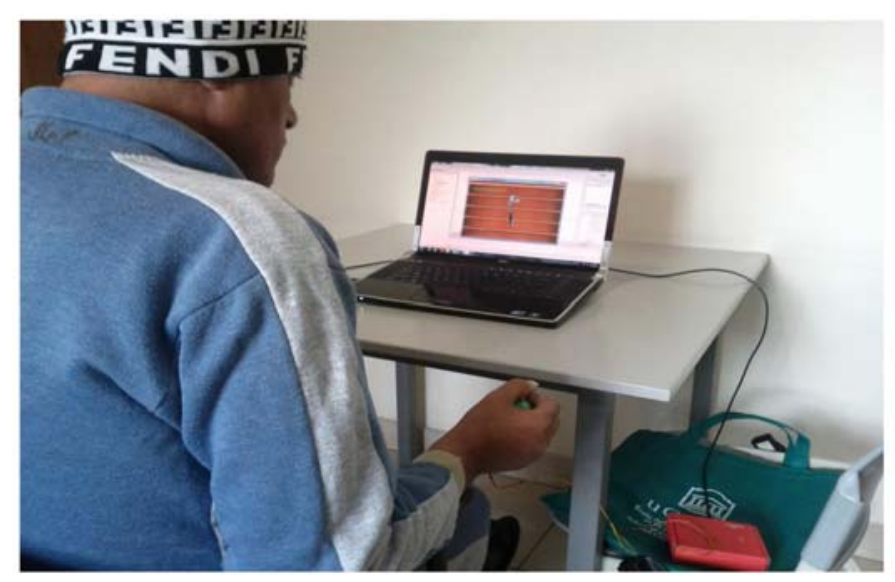

Fig. 7. A patient training with the system

\subsection{Evaluation Set Up}

The evaluations took place at a rehabilitation and senior home complex in the city of Tripoli in Lebanon in collaboration with Halimi and Kabbara physiotherapy center located in the same city. Consent forms were signed prior to any tests. The tests were conducted in 10 sessions over the period of three weeks. The length of each session varied between 15 and 30 minutes depending on the physical and psycho-physiological state of the patient, as well as the recommendations of the therapist who was present during the experiments. We have used a Pentium 4 Dell laptop to run our CAHR framework. Each patient was asked to sit on a chair and rest his hand on the seat's arm and try to be as steady as possible during the exercise. Figure 7 shows one of the patients while training with the Cup \& Plate game.

\subsection{Baseline Evaluation}

At the beginning of each session, patients were provided with a short questionnaire that aims to rate their physiological and psychological state. In order to properly confirm the physiological and psychological condition of the patients, the one method is to ask them to rate their conditions. Such a method would eliminate to attach any sensory devices on the 
patients' body and guarantee a very accurate assessment of their psychophysiological conditions. The patients were simply asked to rate their fatigue and stress levels on a scale of 10 so that we can take these values as a baseline of performance in order to implement the initial configurations. Since the status of a patient might change during the training, we have taken updates from the patients every 5 minutes through an electronic questionnaire that requires them to rate their physical and psychological conditions. Then, this information was fed to the system so that the measured matrix can be updated. Prior to the evaluations, patients were also asked to inform us about any particular issues in the training environment that may cause them discomfort during the training. Particularly, they were asked about the room's luminance, ambient noise, and the laptop's screen resolution. Both patients said that they have no problems regarding these issues. Consequently, the environmental factors were simply ignored since they are not correlated with any other context that may affect the training experience of any of the two patients.

The desired Quality of Physical Performance ( $Q P P$ ) index was chosen by the therapist based on his knowledge about the patient. As previously explained, $Q P P$ is the minimum level that the patient should attain in order to consider his/her performance as a good one.

\subsection{Evaluation Metrics and Results}

Our Physical Performance Quantifier discussed in [17] was modeled based on three inputs: task angle $(\theta)$, wrist velocity ( $\dot{\theta}$ ), and jerkiness $(J)$. Therefore, our main goal was to monitor the progress of the patients specifically over these parameters.

\subsubsection{Range of Motion Results}

Figure 8 and 9 reveals the change in the Range of Motion over the Supination and Pronation motions for the two patients. Calculation of improvements or deterioration is based on the regression curves fit to the data.It can be clearly seen that both patients have shown improvement in their Supination and Pronation Range of Motions. Patient AS who had the least deficit on both motions had shown 43\% improvement on the Supination movement and 48\% enhancement on the Pronation. On the other hand, Patient ML has accomplished $48 \%$ improvement on the Supination motion and $26 \%$ on the Pronation. The largest improvement was realized on the Supination motion in Patient ML who has achieved 82 degrees of range and consequently was able to perform almost a full rotation on that motion by the end of the 10th session.

\subsubsection{Wrist Velocity Results}

In order to determine if there is any improvement in terms of wrist Velocity, we consider three data samples collected during three different sessions for every patient on each motion and study their behaviors with respect to the norms found in [12]. We limited our studies to 3 samples only because it is very inconvenient to graphically display all the results on one curve.

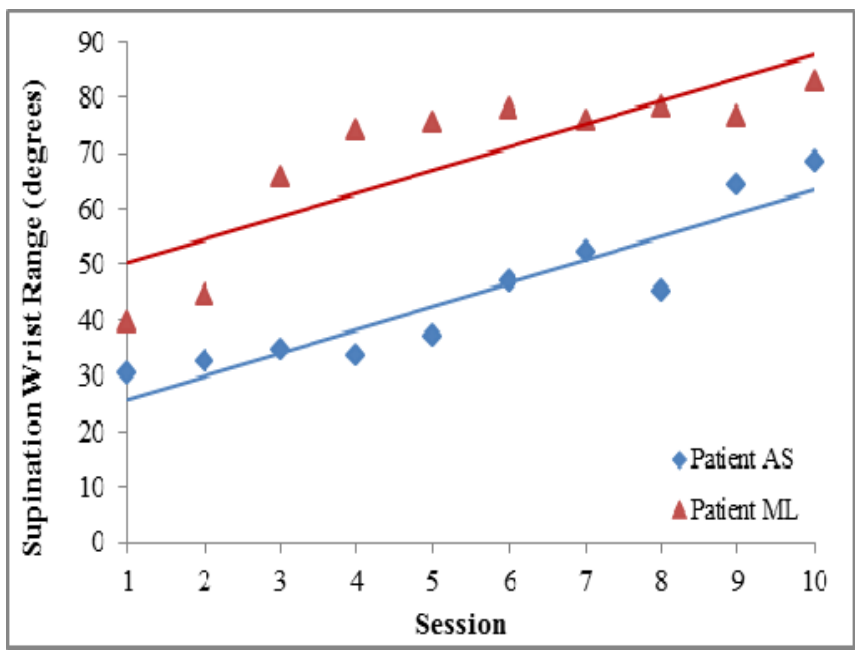

Fig. 8. Range of Motion achieved on the Supination motion

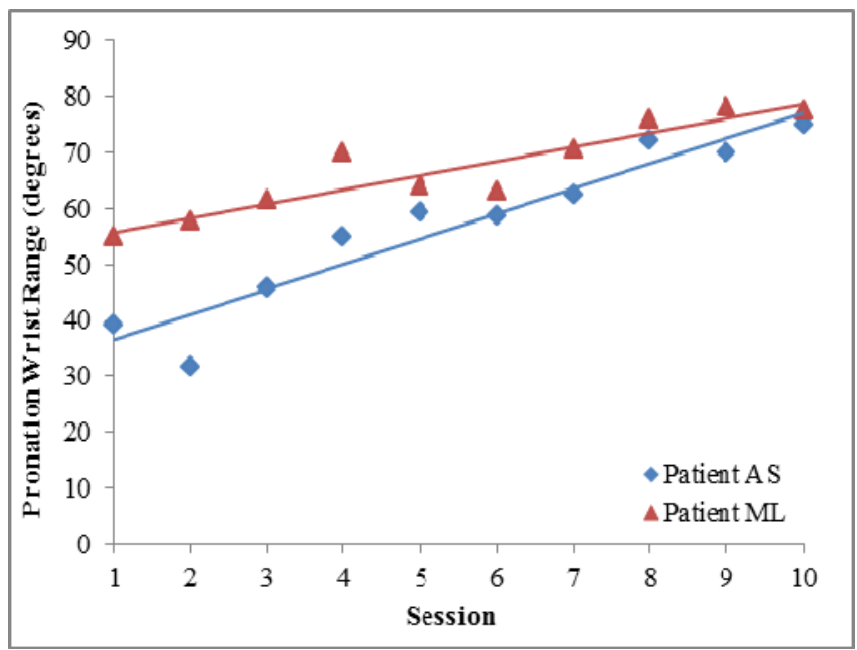

Fig. 9. Range of Motion achieved on the Pronation motion

Figures 10 shows the velocity data collected during session 3, 6, and 9 for both patients while performing the Supination and Pronation set of tasks. The solid black curve represents the Velocity Benchmark Equation found in [12]. Therefore, in order to notice that a progress in performance, the velocity pattern(s) of the session(s) should either converge to the threshold curve or even exceed it. In order to determine the best performance among overlaping curves, we compute the area under each of them. Table 1 presents the area corresponding to each session on the Supination and Pronation motion for each of the patients. The area under the curve can be therefore interpreted as the score that the patient has achieved after the training. Consequently, this would facilitate our analysis since the performances in the sessions could be simply compared with crisp values. 


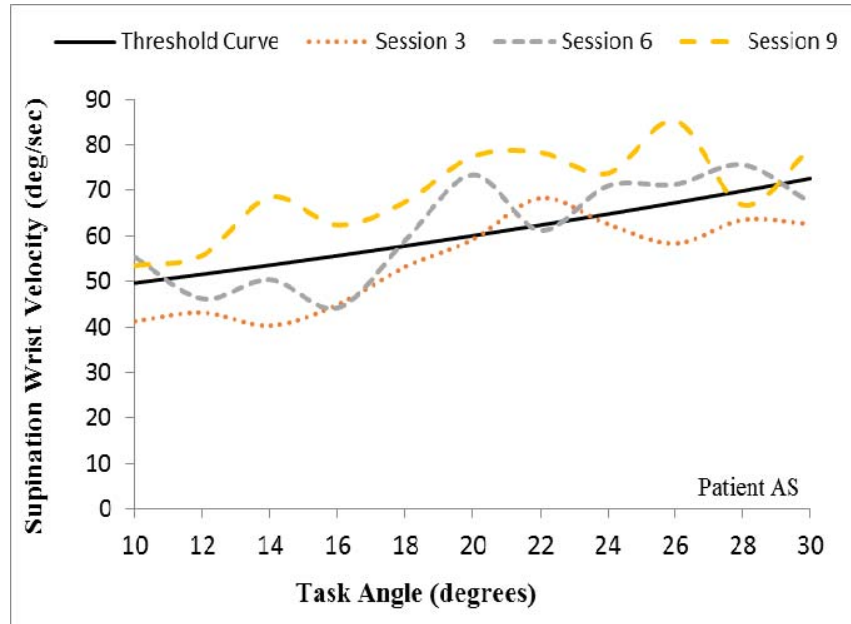

(a)

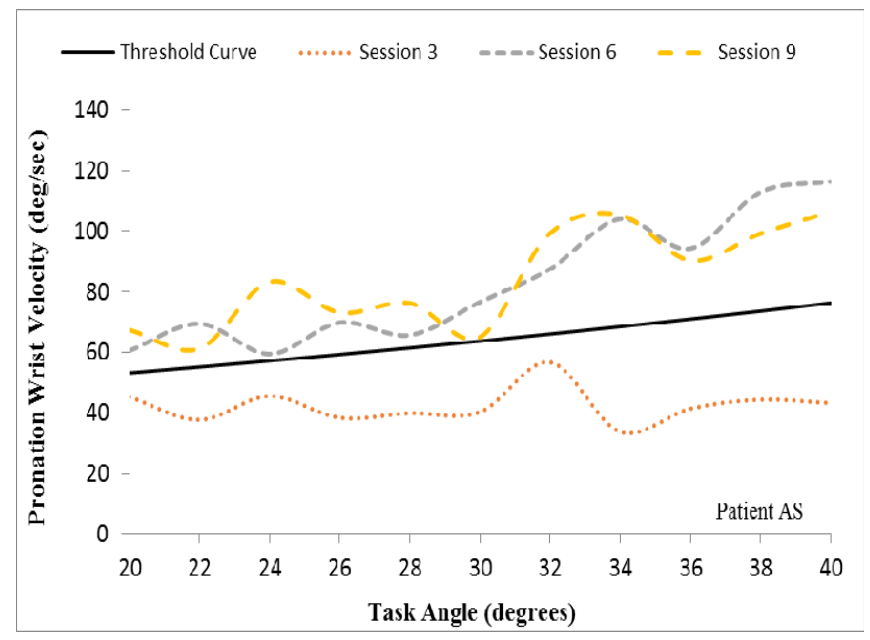

(c)

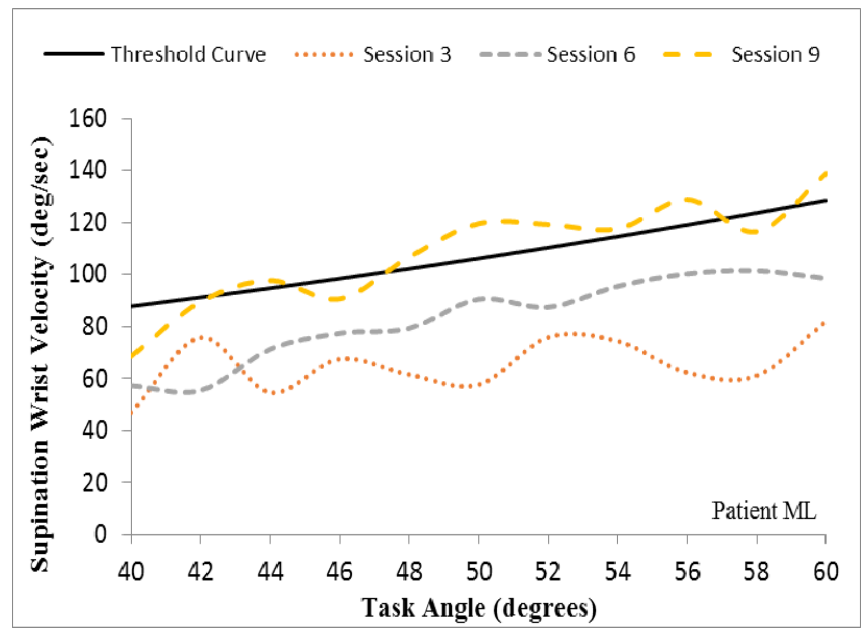

(b)

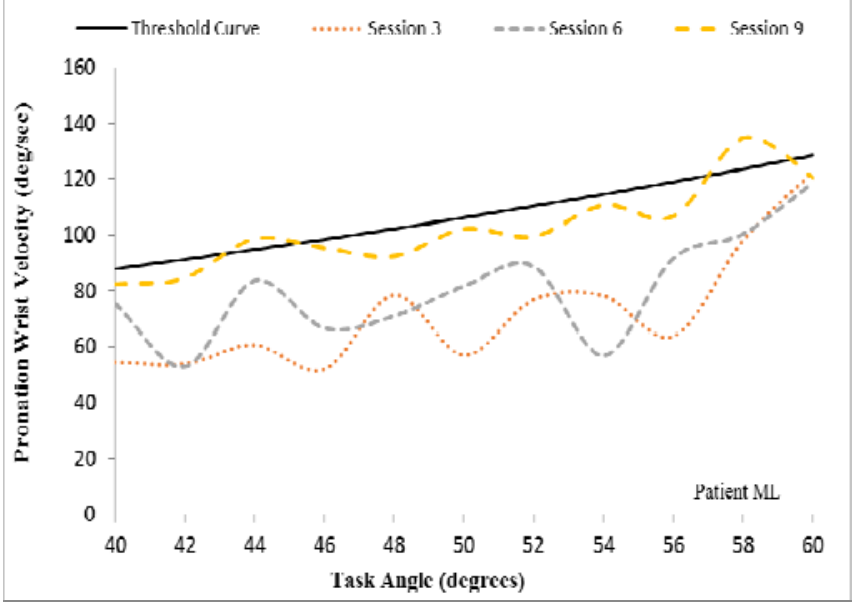

(d)

Fig. 10. The Supination and Pronation velocity patterns for patient AS ,and Patient ML

For Patient AS, we chose velocity data samples for task angles that range between 10 and 30 degrees. It can be deduced from Figure 10 (a) and Table 1 that the patient's performance on the Supination motion started to converge to the threshold by the end of Session 3 and clearly surpassed it by end of Session 10. In the case of Patient ML, we assess his performance based on velocity data samples for task angles that range between 40 and 60 degrees. The results in Figure 10 (b) show that Patient ML's performance on the Supination motion was below the threshold for both Sessions 3 and 6 but then managed to fluctuate around the threshold by the end of Session 10.

The curves in Figure 10 (c) and (d) and the data in Table 1 reveals that Patient AS was almost 33\% below the threshold in Session 3. However, Session 6 and 10 shows around 96\% improvement over Session 3. However, Patient ML's improvement was relatively slow. Even though Figure 10 (b) shows that ML's performance was improving over the sessions, his progress did not reach an acceptable level until Session 9 where he was only 4\% away from the threshold.

TABLE 1

THE AREAS UNDER THE CURVES PRESENTED IN FIGURE 10

\begin{tabular}{|c|c|c|c|c|c|}
\hline & & \multicolumn{4}{|c|}{ Area Under Curve } \\
\hline \multirow{3}{*}{ 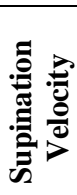 } & Patient & $\begin{array}{c}\text { Session } \\
3\end{array}$ & $\begin{array}{c}\text { Session } \\
6\end{array}$ & $\begin{array}{c}\text { Session } \\
10\end{array}$ & Threshold \\
\hline & AS & 1198 & 1353 & 1537 & 1331 \\
\hline & ML & 1437 & 1831 & 2386 & 2353 \\
\hline$\equiv$ & AS & 935 & 1833 & 1858 & 1412 \\
\hline 焉 & ML & 1588 & 1772 & 2558 & 2354 \\
\hline
\end{tabular}




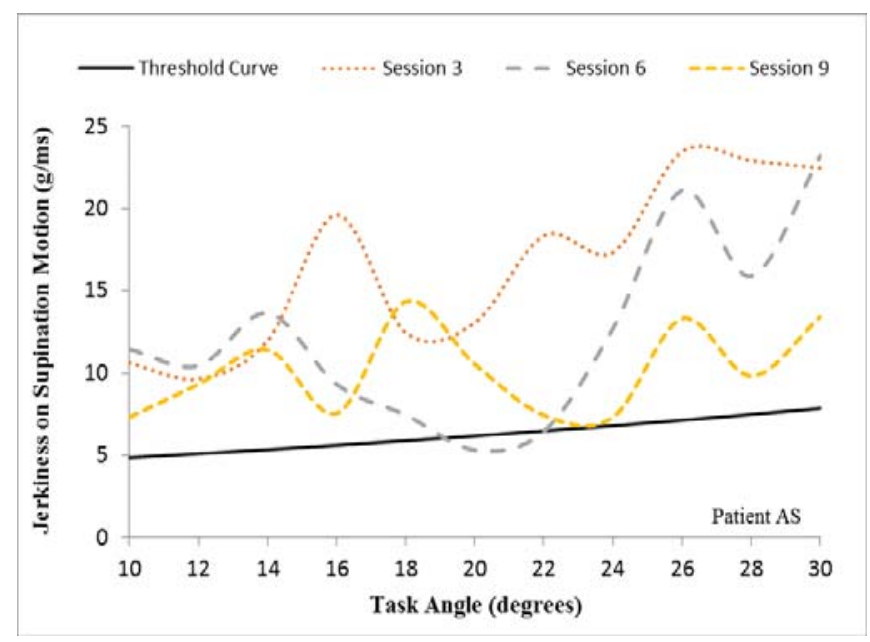

(a)

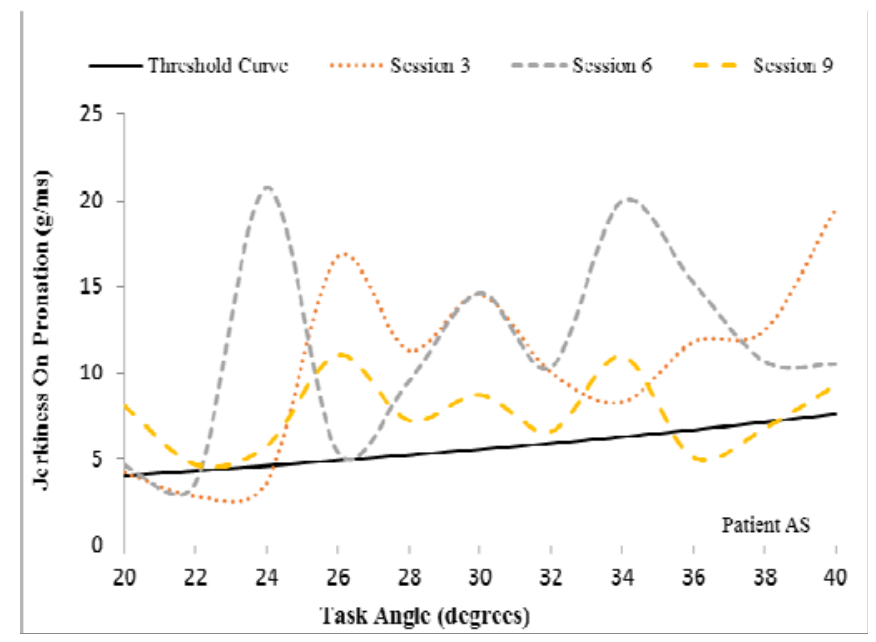

(c)

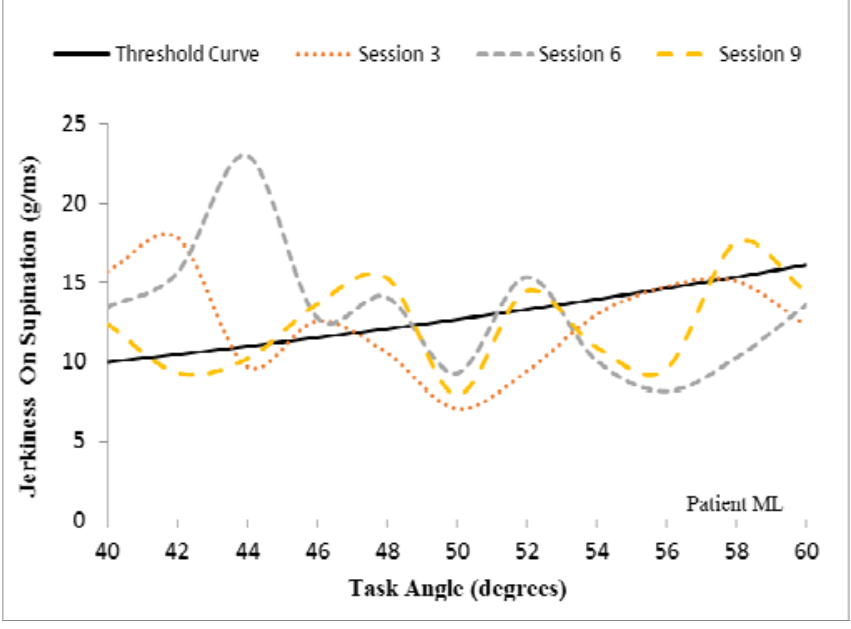

(b)

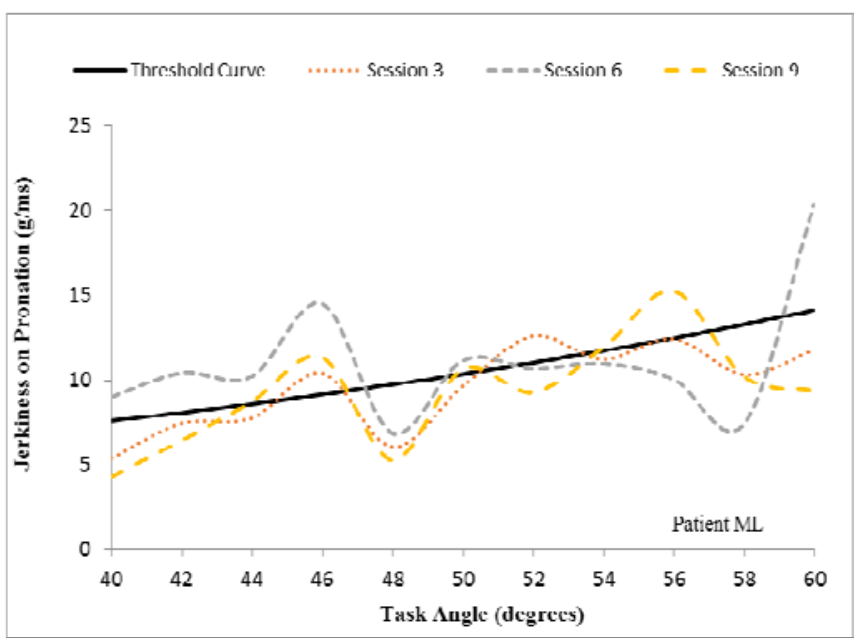

(d)

Fig. 11. The Supination and Pronation jerkiness patterns for patient AS ,and patient ML

\subsubsection{Wrist Jerkiness Results}

The analysis of the wrist Jerkiness on both motions was done based on the same data samples that were used to analyze the velocity. The threshold curves for the Supination and Pronation motions were computed based on the Jerkiness Benchmark Equation found in [12]. Jerkiness is defined as the rate of smoothness of velocity. Therefore, the smaller the value of Jerkiness the smoother the hand velocity. Consequently, a patient performance is considered to be improving if the pattern of Jerkiness is either converging to the threshold curve or go below it (i.e in this case the performance is better than the target threshold level). In terms of the area under the curve presented in Table 2, the area of a certain Jerkiness performance curve should be less than or equal to the area below the threshold.

Figure 11 reveals the results of the Jerkiness obtained on the Supination and Pronation motion respectively. We can deduce from Figure 11 (a) that Patient AS might need more training sessions before his Supination Jerkiness can improve since even after the $10^{\text {th }}$ session, the patient did not achieve a decent progress. On the contrary, Patient ML did not have a problem with his Supination Jerkiness performance. We can clearly observe that his performance over the various sessions is below the threshold level. This can be easily realized from Patient ML's Supination Jerkiness under curve areas presented in Table 2.

Now looking at the Pronation results of both patients, we realize that Patient AS had shown some improvement in Session 9 over the past sessions (i.e. see Figure 11(c)); however, the results did not reach the expected thresholds which means that the patient might need more sessions before optimals results are reached for this metric. On the other hand, Patient ML had no problems with his Pronation Jerkiness throughout the various sessions. Figure 11 (d) shows that the curves were always fluctuating smoothly around the threshold curve. 
TABLE 2

THE AREAS UNDER THE CURVES PRESENTED IN FIGURE 11

\begin{tabular}{|c|c|c|c|c|c|}
\hline & & \multicolumn{4}{|c|}{ Area Under Curve } \\
\hline \multirow{3}{*}{ 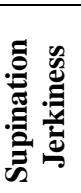 } & Patient & $\begin{array}{c}\text { Session } \\
3\end{array}$ & $\begin{array}{c}\text { Session } \\
6\end{array}$ & $\begin{array}{c}\text { Session } \\
10\end{array}$ & Threshold \\
\hline & AS & 364.02 & 274.12 & 223.89 & 137.4 \\
\hline & ML & 275.30 & 290.90 & 271.80 & 282.25 \\
\hline & AS & 231.20 & 251.10 & 168.89 & 125.04 \\
\hline لَّ & ML & 210.24 & 243.70 & 205.66 & 232.44 \\
\hline
\end{tabular}

\section{CONCLUSION AND FUTURE WORK}

Task adaptation is a crucial part of any virtural rehabilitation system since it can shorten the therapy duration by offering the patient proper training tasks that fit his or her condition. In order to offer a therapy training that can properly adjust to the performance of the patient, the adaptation should not be based only on the patient's physical state, but also on his/her psychophysilogical and enviornmental conditions. In this paper, we have presented an adaptative framework that considers various contextual information to adjust the training environment so that appropriate tasks, and media outputs are provided to the patient. Simulations of the proposed adaption scheme have shown that it can properly cope with various performance patterns. In addition, clinical trials performed with a prototype that is implemented based on the proposed framework have shown an improvement in the performance kinematics of two patients with wrist impairments. Our future work will be focused on finding reliable techniques that can help us detect the fatigue and stress levels in an online fashion during the training.

\section{REFERENCES}

[1] J. Zhou, F. Malric, S. Shirmohammadi, "A new hand-measurement method to simplify calibration in cyberglove-based virtual rehabilitation", IEEE Transactions on Instrumentation and Measurement, 59(10), pp.2496-2504, 2010.

[2] J. Halton, "Virtual rehabilitation with video games: A new frontier for occupational therapy", Occupational Therapy Now, 10(1), pp. 12-14, 2008.

[3] Chen Y., H. Huang, W. Xu, R. I. Wallis, H. Sundaram, T. Rikakis, T. Ingalls, L. Olson, and J. He, "The design of a real-time, multimodal biofeedback system for stroke patient reha-bilitation”, In Proceedings of the 14th annual ACM International Conference on Multi-media, pp. 763-772, 2006.

[4] Alamri A., M. Eid, R. Iglesias, S. Shirmohammadi, and A. El Saddik, "Haptic virtual rehabil-itation exercises for poststroke diagnosis", IEEE Transactions on Instrumentation and Measurement, 57(9), pp. 1876-1884, 2008.

[5] A. Alamri, J. Cha, and A. El Saddik, "AR-REHAB: An augmented reality framework for poststroke-patient rehabilitation", IEEE
Transactions on Instrumentation and Meaurement, 59(10), pp.25542563, 2010.

[6] M. Borghetti, E. Sardini, M. Serpelloni, "Sensorized glove for measuring hand finger flexion for rehabilitation purposes", IEEE Transactions on Instrumentation and Measurement, 62(12), pp.33083314, 2013.

[7] Burke J., M. McNeill, D. Charles, P. Morrow, J. Crosbie, and S. McDonough, "Optimising engagement for stroke rehabilitation using serious games", The Visual Computer, 25(12), pp. 1085-1099, 2009.

[8] Bakkes S., P. Spronck, and J. van den Herik, "Rapid and reliable adaptation of video game AI", IEEE Transactions on Computational Intelligence and Ambient Intelligence in Games, 1(2), pp. 93-104, 2009.

[9] Spronck P., M. Ponsen, I. Sprinkhuizen-Kuyper, and E. Postma,"Adaptive game AI with dy-namic scripting”, Machine Learning, 63(3), pp. 217-248, 2006.

[10] Burdea G., "Virtual Rehabilitation-Benefits and Challenges", Methods of Information in Medicine, 42(5), pp. 519-523, 2003.

[11] Chen Y., W. Xu, H. Sundaram, T. Rikakis, and S. Liu, "Media adaptation framework in bio-feedback system for stroke patient rehabilitation", International Multimedia Conference: Proceedings of the 15 th international conference on Multimedia, 25(9), pp. 47-57, 2007.

[12] A. Karime, M. Eid, W. Gueaieb, and A. El Saddik, "Determining Wrist Reference Kinematics Using a Sensory-Mounted Stress Ball", IEEE International Symposium on Robotics and Sensors Environments, Magdeburg, 2012.

[13] Pirovano M., R. Mainetti, G. Baud-Bovy, P. L. Lanzi, and N. A. Borghese, "Self-Adaptive Games for Rehabilitation at Home", IEEE Computational Intelligence and Games, pp.179-186, 2012

[14] Rossol N., I. Cheng, W. F. Bischof, and A. Basu, "A framework for adaptive training and games in virtual reality rehabilitation environments.", In Proceedings of the 10th Interna-tional Conference on Virtual Reality Continuum and Its Applications in Industry, pp. 343-346, 2011.

[15] Alamri A., J. Cha, and A. El Saddik, "AR:REHAB: an augmented reality framework for poststroke-patient rehabilitation”, IEEE Transactions on Instrumentation and Measurement, 59(10), pp. 25542563, 2010.

[16] A. B. Watson, and D. G. Pelli, "Quest: a bayesian adaptive psychometric method”, Perception and Psychophysics, 33(2), pp. 113-120, 1983

[17] Ali Karime, Mohamad Eid, Jihad Mohamad Aljaam, Abdulmotaleb El Saddik and Wail Gueaieb, "A Fuzzy-based Adaptive Rehabilitation Framework for Home-based Therapy", IEEE Transactions on Instrumentation and Measurement , 63(1), pp.135-144, 2014.

[18] Ma M., M. McNeill, D.Charles, S. McDonough, J.Crosbie, L. Oliver, and C. McGoldrick. "Adaptive virtual reality games for rehabilitation of motor disorders", Universal Access in Human-Computer Interaction, Ambient Interaction, Springer, Vol. 4555, pp. 681-690, 2007.

[19] Chen Y., W. Xu, H. Sundaram, T. Rikakis, and S. Liu, “A Dynamic Decision Network Framework for Online Adaptation in Stroke Rehabilitation”, ACM Transactions on Multimedia Computing, Communications, and Applications (TOMCAPP), 5(1), 2008.

[20] Chen Y., H. Huang, W. Xu, R. I. Wallis, H. Sundaram, T. Rikakis, T. Ingalls, L. Olson, and J. He, "The design of a real-time, multimodal biofeedback system for stroke patient rehabilitation”, In Proceedings of the 14th annual ACM International Conference on Multimedia, pp. 763-772, 2006.

[21] S. Hygge, and I. Knez, "Effects of noise, heat, and indoor lighting on the cognitive performance and self-reported affect”, Journal of Environmental Psychology, 21(3), pp. 291-299, 2001.

[22] Matthews G., L. Dorn, T. W. Hoyes, D. Roy Davies, A. Ian Glendon, and R. G. Taylor, "Driver stress and performance on a driving simulator", The Journal of Human Factors and Ergonomics Society, 40(1), pp. 136-149, 1998.

[23] Driskell J. E., and E. Salas, "Stress and human performance", Lawrence Erlbaum Associates, 1996. 
[24] G. Patsis, H. Sahli, W. Verhelst, and O. De Troyer, "Evaluation of attention levels in a Tetris game using a brain Computer Interface", User Modeling, Adaptation, and Personalization, Springer Berlin Heidelberg, pp. 127-138, 2013

[25] S. Park,.H. Sim, and W. Lee, "Dynamic game difficulty control by using EEG-based emotion recognition”, International Journal of Control \& Automation, 7(3) pp.267-272, 2014.

[26] T.D. Parsons, and J. L. Reinebold,"Adaptive virtual environments for neuropsychological assessment in serious games", IEEE Transactions on Consumer Electronics , 58(2), pp.197-204, 2012.

[27] G. Chanel, C. Rebetez, M. Betrancourt, and T. Pun, "Emotion assessment from physiological signals for adaptation of game difficulty," IEEE Transactions on Systems, Man and Cybernetics, Part A: Systems and Human, 41(6), pp. 1052-1063. 2011.

[28] C. Liu, P. Agrawal, N. Sarkar, and S. Chen, "Dynamic difficulty adjustment in computer games through real-time anxiety-based affective feedback", International Journal of Human-Computer Interaction, 25(6), pp. 506-529, 2009. 